\title{
Measurement of $\mathbf{D}^{*}$ Production in Diffractive Deep Inelastic Scattering at HERA
}

\section{Karel Černý*}

Institute of Particle and Nuclear Physics, Charles University, Prague

E-mail: kcerny@ipnp.troja.mff.cuni.cz

Measurements of $D^{*}(2010)$ meson production in diffractive deep inelastic scattering $\left(5<Q^{2}<\right.$ $100 \mathrm{GeV}^{2}$ ) are presented which are based on HERA data recorded at a centre-of-mass energy $\sqrt{s}=319 \mathrm{GeV}$ with an integrated luminosity of $287 \mathrm{pb}^{-1}$ collected by the H1 detector. The reaction $e p \rightarrow e X Y$ is studied, where the system $X$, containing at least one $D^{*}(2010)$ meson, is separated from a leading low-mass proton dissociative system $Y$ by a large rapidity gap. The kinematics of $D^{*}$ candidates are reconstructed in the $D^{*} \rightarrow K \pi \pi$ decay channel. The measured cross sections compare favourably with next-to-leading order QCD predictions, where charm quarks are produced via boson-gluon fusion and they are independently fragmented to the $D^{*}$ mesons. The calculations rely on the collinear factorisation theorem and are based on diffractive parton densities previously obtained by $\mathrm{H} 1$ from fits to inclusive diffractive cross sections. The data are further used to determine the diffractive to inclusive $D^{*}$ production ratio in deep inelastic scattering.

XXV International Workshop on Deep-Inelastic Scattering and Related Subjects

3-7 April 2017

University of Birmingham, UK

\footnotetext{
${ }^{*}$ Speaker.

${ }^{\dagger}$ On behalf of the H1 Collaboration.
} 


\section{Introduction}

The collinear factorisation [1] approach is an effective tool to describe the diffractive deep inelastic scattering (DDIS) processes $e p \rightarrow e X Y$, where the systems $X$ and $Y$ are separated by a large gap in rapidity due to the colourless exchange, often referred to as a pomeron $(\mathbb{P})$. To date, analyses of HERA data support the validity of the collinear factorisation theorem in DDIS as evidenced by experimental results on inclusive production, dijet production and $D^{*}$ production. A brief account of a new measurement of $D^{*}(2010)$ meson production in DDIS [2] is presented here. The $D^{*}$ meson originates from the fragmentation of a charm quark, produced mainly via the boson-gluon-fusion $\left(\gamma^{*} g \rightarrow c \bar{c}\right)$ at HERA energies, figure 1, and is therefore sensitive to the gluon content of the pomeron. Compared to the previous $\mathrm{H} 1$ publication [3] the analysis presented corresponds to a sixfold increase in the integrated luminosity.

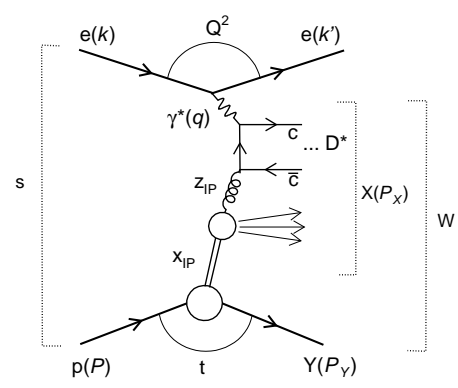

Figure 1: The leading order diagram for open charm production in DDIS at HERA in the picture of collinear and proton vertex factorisation.

\section{Kinematics of the deep inelastic scattering}

The standard DIS kinematics is described in terms of the well known invariants

$$
s=(k+P)^{2}, \quad Q^{2}=-q^{2}, \quad y=\frac{q \cdot P}{k \cdot P}, \quad W^{2}=(q+P)^{2}, \quad x=\frac{Q^{2}}{2 q \cdot P},
$$

where the four-vectors are indicated in figure 1. The diffractive kinematics are defined as:

$$
M_{X}^{2}=\left(P_{X}\right)^{2}, \quad M_{Y}^{2}=\left(P_{Y}\right)^{2}, \quad t=\left(P-P_{Y}\right)^{2}, \quad x_{\mathbb{P}}=\frac{q \cdot\left(P-P_{Y}\right)}{q \cdot P}, \quad z_{\mathbb{P}}=\frac{\hat{s}+Q^{2}}{M_{X}^{2}+Q^{2}},
$$

where $M_{X}$ and $M_{Y}$ are the invariant masses of the systems $X$ and $Y$, respectively, $t$ is the squared four-momentum transfer at the proton vertex and $x_{\mathbb{P}}$ the fraction of the proton's longitudinal momentum transferred to the system $X$ and $z_{\mathbb{P}}$ represents, in leading order, the momentum fraction of $\mathbb{P}$ participating in the $\gamma^{*} g \rightarrow c \bar{c}$ process, where $\hat{s}$ denotes its centre-of-mass energy squared.

\section{Monte Carlo Models and Fixed Order QCD Calculations}

The diffractive proton elastic $e p \rightarrow e X\left(D^{*}\right) p$ and proton dissociative $e p \rightarrow e X\left(D^{*}\right) Y$ are modelled with the RAPGAP Monte Carlo event generator [4]. Following a detailed H1 detector response simulation the samples are passed through the same analysis chain as used for data and are used to correct the data for detector effects.

Predictions for $D^{*}$ cross sections in next-to-leading-order (NLO) QCD precision are obtained using HVQDIS [5, 6]. The calculation relies on collinear factorisation using H1 2006 DPDF Fit B NLO parton density functions [7]. Massive charm quarks are produced via $\gamma^{*}$-gluon fusion. Fragmentation into $D^{*}$ mesons is performed independently in the $\gamma^{*} p$ rest frame using the Kartvelishvili 
parameterisation with parameter values, as well as the uncertainties on them, suited for use with HVQDIS [8]. The factorisation and renormalisation scales are set to $\mu_{r}=\mu_{f}=\sqrt{Q^{2}+4 m_{c}^{2}}$ with the value $m_{c}=1.5 \mathrm{GeV}$ for the charm pole mass. The variations by factors of 0.5 and 2 are used as the uncertainty of the scale's choice. The uncertainty introduced by the particular choice of $m_{c}$ is evaluated by varying it to $1.3 \mathrm{GeV}$ and $1.7 \mathrm{GeV}$.

\section{Experimental technique}

A detailed description of the $\mathrm{H} 1$ detector can be found elsewhere [9]. The selection of DIS events is ensured by a scattered electron signal in the backward lead-scintillating fiber calorimeter. The decay products of the $D^{*}$ meson are observed as tracks in the central tracker. Hadronic final state objects represent combined information from central tracker and LAr calorimeter.

The measured $Q^{2}$ and $y$ ranges are defined as $5<Q^{2}<100 \mathrm{GeV}^{2}$ and $0.02<y<0.65$, respectively. The large rapidity gap (LRG) selection of diffractive events is primarily provided by a requirement on the position of the most forward cluster in the LAr calorimeter above the $800 \mathrm{MeV}$ energy threshold, $\eta_{\max }<3.2$ augmented by vetoes from forward detectors. The $x_{\mathbb{P}}<0.03$ range is used. The detection of $D^{*}$ mesons is based on the full reconstruction of its decay products in the 'golden channel': $D^{*+} \rightarrow D^{0} \pi_{\text {slow }}^{+} \rightarrow\left(K^{-} \pi^{+}\right) \pi_{\text {slow }}^{+}+(C . C$.) with a branching ratio of $\sim 2.7 \%$. The $K$ and $\pi$ candidate tracks are required to satisfy $p_{t}>0.3 \mathrm{GeV}$ transverse momentum cuts while the $\pi_{\text {slow }}$ track $p_{t}>0.12 \mathrm{GeV}$ is required in the laboratory frame. An $80 \mathrm{MeV}$ mass window cut ensures consistency with $D^{0}$ hypothesis of $K$ and $\pi$ system. The $D^{*}$ meson candidate kinematics is restricted to $p_{t, D^{*}}>1.5 \mathrm{GeV}$ and $\left|\eta_{D^{*}}\right|<1.5$. The variable $\Delta m=m\left(K^{\mp} \pi^{ \pm} \pi_{\text {slow }}^{ \pm}\right)-m\left(K^{\mp} \pi^{ \pm}\right)$ is used to determine the $D^{*}$ signal by means of simultaneous fits of the right and wrong charge combinations of the tracks for a better background shape determination yielding $N\left(D^{*}\right)=1169 \pm$ 58. The $N\left(D^{*}\right)$ obtained from fits to the data in bins of event observables are found to compare well with the simulation.

\section{Results}

The number of fitted $D^{*}$ mesons is corrected for detector effects, branching ratio of the golden channel, contribution of other $D^{*}$ decays and higher order QED processes at the lepton vertex. The phase space of the measurement reads $5<Q^{2}<100 \mathrm{GeV}^{2}, 0.02<y<0.65, x_{\mathbb{P}}<0.03$, $p_{t, D^{*}}>1.5 \mathrm{GeV}$ and $\left|\eta_{D^{*}}\right|<1.5$. Since $M_{Y}$ and $t$ are not measured directly an extrapolation is performed to the range of $M_{Y}<1.6 \mathrm{GeV}$ and $|t|<1 \mathrm{GeV}^{2}$. The integrated cross section of $D^{*}$ production is measured to be $\sigma_{e p \rightarrow e Y X\left(D^{*}\right)}=314 \pm 23$ (stat.) \pm 35 (syst.) pb. The theoretical value calculated in next-to-leading order QCD reads $\sigma_{e p \rightarrow e Y X\left(D^{*}\right)}^{\text {theory }}=265_{-40}^{+54}(\text { scale })_{-54}^{+68}\left(\mathrm{~m}_{\mathrm{c}}\right)_{-8.2}^{+7.0}($ frag. $)$ ${ }_{-35}^{+31}(\mathrm{DPDF}) \mathrm{pb}$. Within uncertainties both normalisation and shapes of the measured cross sections are reproduced by the theory, see figure 2. The experimental precision in the region of medium and highest $x_{\mathbb{P}}$ and $z_{\mathbb{P}}$, figure $2 \mathrm{~b}$, may provide a room further constraints on the gluon part of the DPDFs.

The fraction of diffractive contribution to the $D^{*}$ production in DIS, $R_{D}$, is determined using also the H1 DIS results [10]. Integrated over the whole phase space the results read $R_{D}=6.6 \pm$ 0.5 (stat) ${ }_{-0.8}^{+0.9}($ syst $) \%$ and $R_{D}^{\text {theory }}=6.0_{-0.7}^{+1.0}(\text { scale })_{-0.4}^{+0.5}\left(m_{c}\right)_{-0.8}^{+0.7}(\mathrm{DPDF})_{-0.04}^{+0.02}($ frag $) \%$ for the data 

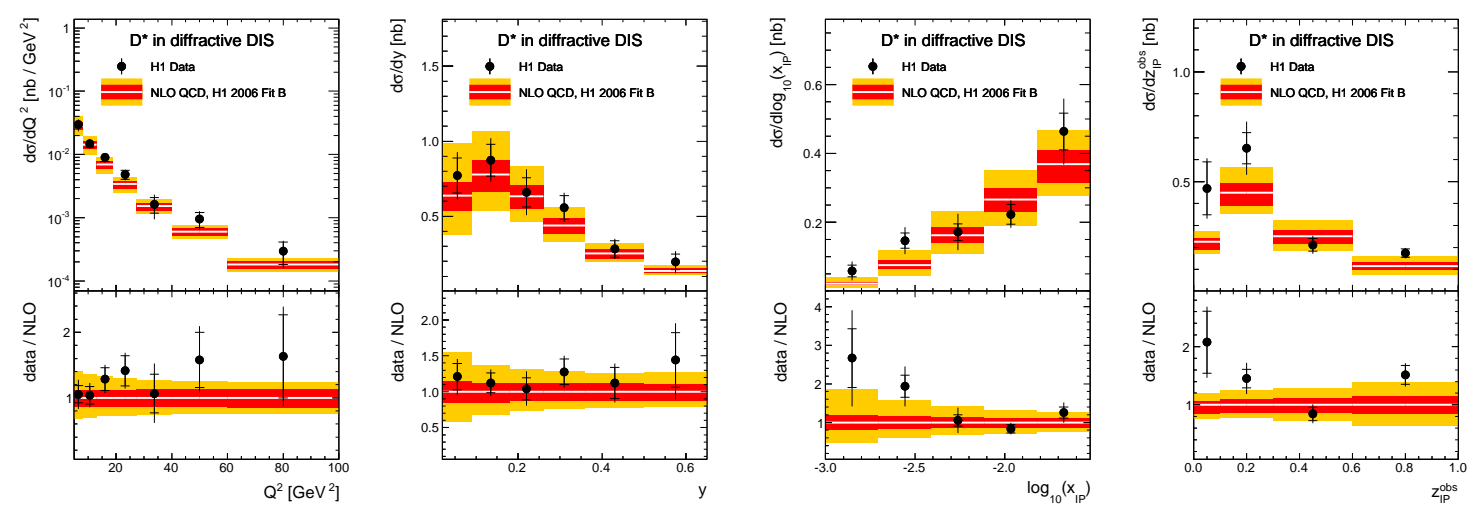

(a)

(b)

Figure 2: Differential $D^{*}$ production cross sections in DDIS and data to theory ratios as a function of; (a) $Q^{2}$ and $y$, (b) $\log _{10}\left(x_{\mathbb{P}}\right)$ and $z_{\mathbb{P}}^{o b s}$.

and theoretical prediction, respectively, where the predictions profit from partial cancellation of the scale and $m_{c}$ uncertainties. The fractions measured differentially are shown in figure $3 \mathrm{a}$. Both the total and the differential data $R_{D}$ are consistent with theory showing strong kinematical dependence that can be explained limitations of the diffractive phase space domain. In figure $3 \mathrm{~b}, R_{D}$ integrated over the full phase space is compared with previous measurements performed at HERA both in the DIS regime [11-13] and in photoproduction [14].
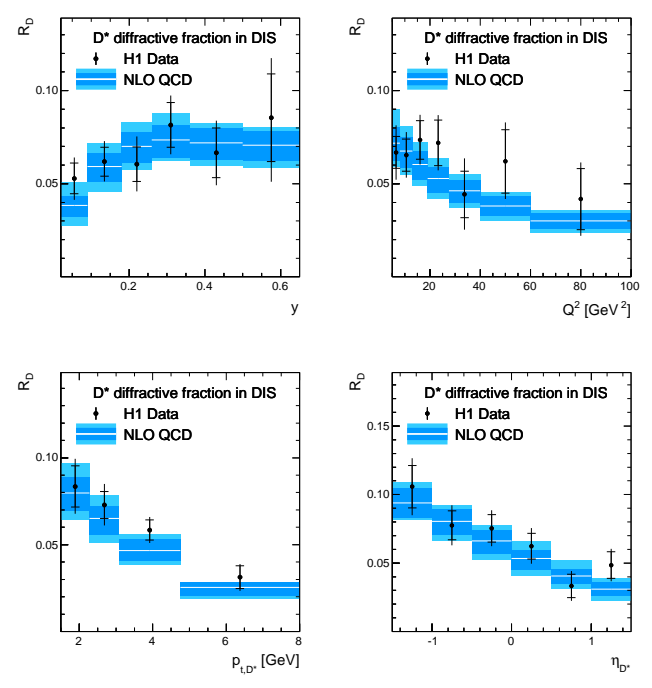

(a)

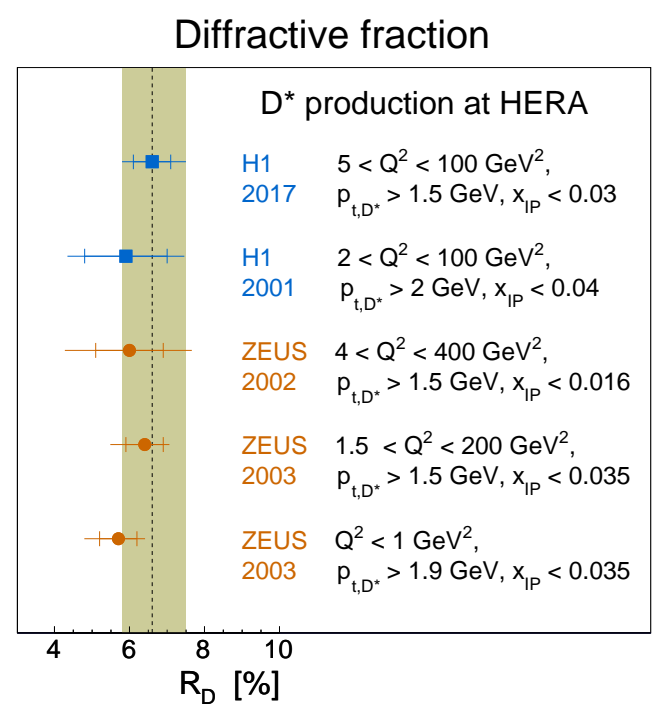

(b)

Figure 3: The diffractive fraction $R_{D}$ as a function of $y, Q^{2}, p_{t, D^{*}}$ and $\eta_{D^{*}}$ (a). Integrated $R_{D}$ compared with previous measurements from HERA (b). 


\section{Conclusions}

The integrated and differential cross sections of $D^{*}(2010)$ production in diffractive deep inelastic scattering are measured. The measured cross sections are well described by theoretical predictions in next to leading order QCD. This supports the validity of collinear factorisation.

The measured diffractive fraction of $D^{*}$ production cross section in deep inelastic scattering is in agreement with theoretical predictions in next-to-leading order QCD. The value of the diffractive fraction is found to depend on certain observables. It is, however, observed to be largely independent of other details of the phase space definition.

\section{References}

[1] J. C. Collins, Proof of factorization for diffractive hard scattering, Phys. Rev. D57 (1998) 3051-3056, [hep-ph/9709499].

[2] H1 collaboration, V. Andreev et al., Measurement of $D^{*}$ production in diffractive deep inelastic scattering at HERA, Eur. Phys. J. C77 (2017) 340, [1703.094 76].

[3] H1 collaboration, A. Aktas et al., Diffractive open charm production in deep-inelastic scattering and photoproduction at HERA, Eur. Phys. J. C50 (2007) 1-20, [hep-ex/ 0610076$].$

[4] H. Jung, Hard diffractive scattering in high energy ep collisions and the Monte Carlo Generator RAPGAP, Comp. Phys. Comm. 86 (1995) 147 - 161.

[5] B. W. Harris and J. Smith, Heavy quark correlations in deep inelastic electroproduction, Nucl. Phys. B452 (1995) 109-160, [hep-ph/9503484].

[6] B. W. Harris and J. Smith, Charm quark and $D^{* \pm}$ cross-sections in deeply inelastic scattering at HERA, Phys. Rev. D57 (1998) 2806-2812, [hep-ph / 9706334$].$

[7] H1 collaboration, A. Aktas et al., Measurement and QCD analysis of the diffractive deep-inelastic scattering cross-section at HERA, Eur. Phys. J. C48 (2006) 715-748, [hep-ex/ 0606004 ].

[8] H1 collaboration, F. D. Aaron et al., Study of Charm Fragmentation into $D^{* \pm}$ Mesons in Deep-Inelastic Scattering at HERA, Eur. Phys. J. C59 (2009) 589-606, [0808 . 1003 ].

[9] H1 collaboration, I. Abt et al., The H1 detector at HERA, Nucl. Instrum. Meth. A386 (1997) 310-347, ibid. 348-396.

[10] H1 collaboration, F. D. Aaron et al., Measurement of $D^{* \pm}$ Meson Production and Determination of $F_{2}^{c \bar{c}}$ at low $Q^{2}$ in Deep-Inelastic Scattering at HERA, Eur. Phys. J. C71 (2011) 1769, [1106.1028].

[11] $\mathrm{H} 1$ collaboration, $\mathrm{C}$. Adloff et al., $D^{* \pm}$ meson production in deep inelastic diffractive interactions at HERA, Phys. Lett. B520 (2001) 191-203, [hep-ex/ 0108047 ].

[12] ZEUS collaboration, S. Chekanov et al., Measurement of diffractive production of $D^{* \pm}(2010)$ mesons in deep-inelastic scattering at HERA, Phys. Lett. B 545 (2002) 244 - 260.

[13] ZEUS collaboration, S. Chekanov et al., Measurement of the open-charm contribution to the diffractive proton structure function, Nucl. Phys. B 672 (2003) 3-35.

[14] ZEUS collaboration, S. Chekanov et al., Diffractive photoproduction of $D^{* \pm}$ (2010) at HERA, Eur. Phys. J. C51 (2007) 301-315. 